\title{
Heat flux evaluation in high temperature ring-on-ring contacts
}

\author{
A. Tavasci ${ }^{1}$, F. Arizzi ${ }^{1,2}$, D. Dini ${ }^{3}$, E. Rizzi ${ }^{2}$ \\ ${ }^{1}$ TenarisDalmine, Italy; ${ }^{2}$ University of Bergamo, Italy; ${ }^{3}$ Imperial College London, UK \\ atavasci@tenaris.com
}

\begin{abstract}
A comprehensive methodology to investigate heat flux in a ring-on-ring tribometer is presented. Thermal fluxes under high contact pressures and temperature differences were evaluated through an experimental campaign and by a numerical procedure of inverse analysis applied to surface temperature measurements. An approximation of a two-dimensional time-dependent analytical solution for the temperature distribution was first developed and subsequently adapted to mimic the specific testing configuration characteristics; the problem was finally simplified to enable further inverse analysis. Experiments were performed using an innovative high temperature ring-on-ring tribometer. The evaluated contact heat-transfer rates were reported as a function of normal load and temperature difference between the discs under steady-state conditions; the results reported here show that, in the present test configuration, the temperature difference has stronger influence than the applied load in terms of heat transfer induced by contact.
\end{abstract}

Key-words: Ring-on-ring tribometer; High temperature; Thermal flux; Heat transfer coefficient; Analytical model; Inverse analysis.

\section{Introduction}

During hot deformation processes, tool surfaces experience severe thermal fluxes which depend on heat conduction. This is, in turn, a function of the surface state, the application of possible lubricants and the presence of surface oxides, as well as of specific contact conditions and temperature differences with the workpiece. In many cases, the transfer of thermal energy is similar in magnitude to the heat transfer generated by frictional forces. Thus, investigations related to this specific topic form a significant research branch within the general realm of tribology [1].

At present, there is a strong industrial need to achieve more accurate, predictive and computer-based models of the underlying physical processes governing high temperature metal-forming processes. However, existing models are somewhat inadequate, mainly due to the inaccurate definition of mechanical and thermal boundary conditions, namely in terms of friction and heat transfer, respectively. For most industrial hot metalforming operations, the complexity of the interaction between tool and workpiece makes the direct evaluation of the evolution of both friction and heat transfer difficult and impractical. Thus, in the absence of a detailed insight and due to a lack of fundamental understanding of the mechanisms of heat transfer at a moving interface, most models assume oversimplified descriptions, or determine an average value of the so-called Heat Transfer Coefficient ( $h t c)$; often this corresponds to evaluating a coefficient that takes into account all 
thermal effects [2], without separating the various contributions from conduction through the actual contact spots, conduction through the interstitial medium, and radiation occurring at the apparent contact area [3]-[6].

In past years, several efforts have been devoted to the understanding of the role played by heat transfer phenomena during hot deformation processes (see e.g. [2], [7]). The availability of complex analytical/numerical models for the process simulation allows the achievement of a more accurate evaluation of the htc based on sophisticated experimental data and the advanced use of procedures of inverse analysis. In particular, the adoption of such solutions in the definition of the objective function in dedicated inverse analysis algorithms shall give rise to a more precise and direct characterization of the htc for in-service operating conditions. However, many inverse analysis methods are based on filling the gap between measurements and output of the model equations [8]-[16]; hence, although these methods may provide more refined parameters, they cannot be easily generalized and they are not suitable for the analysis of the influence of the contact parameters governing the problem under investigation. Despite such efforts, the difficulties of performing laboratory measurements, combined with the complexity of the tool-workpiece interface, result in a wide range of the estimated values of $h t c$ reported in the literature [17].

In the present work, a comprehensive methodology based on combined experimental/analytical analyses is adopted to investigate the global heat flux occurring at the contact region in a ring-on-ring tribometer. The testing device and the associated testing protocols, used to characterize the friction between solids under relative motion, are introduced first. These were employed here to assess heat fluxes in high temperature contacts under pure rolling. Then, a newly-developed methodology implemented to model the heat flux and temperature variation within the system under investigation is presented. Quantitative results for realistic inservice conditions are obtained by means of a final dedicated inverse analysis, matching the temperature measurements with the predictions from the analytical model, specifically tailored for representing heat transfer on a ring-on-ring contact at high temperature.

\section{Material and methods}

Thermal fluxes under high contact pressures and temperature differences were evaluated through a numerical procedure of inverse analysis (discussed in Section 3) applied to surface temperature measurements and developed to produce an accurate estimate of heat transfer at the contact between two components. Experiments were run on a ring-on-ring tribometer, designed to achieve high temperature in the contact between the two rotating discs, in the same range as the temperature experienced during hot deformation processes. The aim was also to control separately the temperature of the two discs. A simplified sketch of the testing configuration and temperature monitoring is illustrated in Figure 1.

\subsection{Materials}

Test discs were machined from bars of carbon steel $(0.40 \% \mathrm{C})$. Two disc dimensions were employed: $120 \mathrm{~mm}$ diameter and $25 \mathrm{~mm}$ wide (large size); $50 \mathrm{~mm}$ diameter and $34 \mathrm{~mm}$ wide (small size). All specimens presented surface finishing from smooth lathe machining (root mean square - r.m.s. - roughness less than $3.2 \mu \mathrm{m}$ ). The testing configuration was obtained by coupling two cylindrical discs of different dimensions. Thermo-physical properties of the material were estimated through the use of a commercial software [18]. 


\subsection{Equipment set-up}

Specimens were mounted on horizontal electrically motorized spindles of $24 \mathrm{~mm}$ diameter and driven independently at controlled speeds. The normal force between the two discs was applied through a pneumatic cylinder. The experimental device was equipped with a main induction heater of $24 \mathrm{~kW}$ power for heating up the larger disc and a second induction heater of $12 \mathrm{~kW}$ power for the smaller one. Coils were located at an effective radial distance for induction heating. Thus, the temperature rises radially from the external hot spot to the spindle. The disc surface temperature evened out during rotation due to the relative motion of the disc with respect to the heat source. Induction power was regulated in a closed loop in order to attain the target value of surface temperature measured through a devoted pyrometer (Figure 1).

\subsection{Experiments}

Long-term tests were carried out at a zero slide/roll ratio, corresponding to pure rolling. Initially the rotating discs were heated up separately and in the absence of contact, to target different surface temperatures for the hot disc and the cold disc. The step duration was enough to let both samples to achieve a steady state condition of temperature distribution. Once the steady state temperature was approached, samples were put in contact under the normal load and the test was run for 30 minutes. At this stage, only the main induction heater was switched on, which guaranteed a constant surface temperature for the hot disc during the whole contact time, whereas the second induction heater was turned off and the surface temperature evolution was recorded for the cold disc. Under these working conditions, the temperature profile of the cold disc evolved due to (i) the heat flux in the contact region, (ii) the radiation from the hot disc, and (iii) the radiation and convection in the surrounding air. The induction heater was excluded to gain more sensitivity towards these variables (uncontrolled fluctuations in power supply could be as relevant as the heat flux).

\subsection{Testing conditions}

Tests were run at fixed disc rotational speed of $60 \mathrm{rpm}$ for the hot disc, in a range of normal loads and initial temperatures, corresponding to 4 different cases, as shown in Table 1. A single test was carried out for each condition once the experimental reproducibility was proven through preliminary investigations covering a wide range of input parameters.

\begin{tabular}{ccccc||}
\hline \multirow{2}{*}{ Normal load } & \multicolumn{2}{c|}{ Initial temperature } & Initial temperature difference & Case \\
\cline { 2 - 5 } & Hot disC & Cold disC & $220{ }^{\circ} \mathrm{C}$ & 1 \\
\multirow{2}{*}{$500 \mathrm{~N}$} & $400^{\circ} \mathrm{C}$ & $180^{\circ} \mathrm{C}$ & $460^{\circ} \mathrm{C}$ & 2 \\
& $800^{\circ} \mathrm{C}$ & $340^{\circ} \mathrm{C}$ & $220^{\circ} \mathrm{C}$ & 3 \\
\multirow{2}{*}{$2000 \mathrm{~N}$} & $400^{\circ} \mathrm{C}$ & $180^{\circ} \mathrm{C}$ & $460^{\circ} \mathrm{C}$ & 4 \\
\hline
\end{tabular}

Table 1: Test conditions.

\subsection{Measurements}

During the contact step, surface temperature evolution due to the rotating contacts was measured at the surface, in the middle of the specimen width, using a pyrometer located at $90^{\circ}$ from the contact region (Figure 1). The pyrometer provided stable data acquisition from $80^{\circ} \mathrm{C}$ to $600^{\circ} \mathrm{C}$. The emissivity was set to a constant value of 0.75 , after comparison with thermocouple measurements. 


\section{Theory}

The inverse analysis procedure used by the authors needed an accurate mathematical description of the physical processes of heat transfer arising in the present experimental configuration. Given the testing procedure to be mimicked and the temperature measurement methodology outlined above, the aim of the mathematical model is to develop a solution of the heat conduction problem in cylindrical coordinates; the contact heat flux was evaluated by focusing on the surface temperature variation in the cold disc, which was modelled as a hollow cylindrical body. The mathematical solution had to be designed to consider the heat transfer due to conduction, convection and radiation (at the present test temperatures the last contribution cannot be neglected). In particular, the external surface underwent a concentrated thermal flux due to the contact with the hot disc on a narrow surface arc segment and a radiation from the hot disc, as well as cooling in the surrounding air by convection and radiation. The internal surface of the cold disk was subjected only to heat exchange with the driven spindle by conduction.

In order to obtain a simple modeling reference for inverse analysis purposes in a reasonable computational time through effective models, an approximation of a three-dimensional time-dependent analytical solution for the temperature distribution calculation was developed to replicate the specific testing configuration. As a first step to achieve this, the real three-dimensional problem was initially simplified using a two-dimensional approximation, which considered the samples as infinitely long. Moreover, because the aim was that of modelling the temperature evolution in the cylinder's cross section, for which the external surface temperature was measured through a pyrometer, the problem was further simplified. A one-dimensional approximation, in which the concentrated heat flux is distributed along the circumference, was introduced to calculate the time evolution of temperature in the radial direction. Indeed, the analysis of the surface temperature profiles (see Section 4.2) confirmed that the temperature measured at $90^{\circ}$ from the contact area (Figure 1) was only mildly affected by the peak value occurring immediately at the end of the contact length.

\subsection{D transient solution}

Omitting the axial coordinate of the cylinder according to the previous assumption, the thermal condition for the cold disc (Figure 2 ) is mathematically expressed by the following complete equation:

$$
\frac{1}{r} \frac{\partial}{\partial r}\left(r \frac{\partial u}{\partial r}\right)+\frac{1}{r^{2}} \frac{\partial^{2} u}{\partial \theta^{2}}=\frac{\omega}{\alpha} \frac{\partial u}{\partial \theta}+\frac{1}{\alpha} \frac{\partial u}{\partial t}
$$

where $u=u(r, \theta, t)$ is the temperature, $t$ the time, $\mathrm{r}$ the radial coordinate, $\theta$ the angular coordinate and $\alpha$ the material thermal diffusivity. Note that the coordinate system $(r, \theta)$ is centred on the axis of the cylinder and it is such that the origin of the polar coordinate $\theta$ corresponds to the concentrated thermal flux due to contact; the ring rotates with constant speed $\omega$ with respect to this coordinate system [19].

Invoking circular symmetry, Eq. ( 1 ) can be reduced to the following one-dimensional formulation:

$$
\frac{1}{r} \frac{\partial}{\partial r}\left(r \frac{\partial u}{\partial r}\right)=\frac{1}{\alpha} \frac{\partial u}{\partial t}
$$


The boundary conditions for the one-dimensional problem in Eq. 2 at the external radius, $R_{e}$, and at the internal radius, $R_{i}$, can be written respectively as (see Figure 2 ):

$$
\begin{gathered}
-k \frac{\partial}{\partial r} u\left(R_{e}, t\right)=q_{a}+q_{r R}+F_{H-C} q_{r H D}+\frac{\varphi_{0}}{2 \pi} q_{c} \\
-k \frac{\partial}{\partial r} u\left(R_{i}, t\right)=q_{i},
\end{gathered}
$$

where $k$ is the thermal conductivity, $q_{a}$ is the heat exchanged by convention in air, $q_{r R}$ is the radiation heat exchanged with the environment, $q_{r H D}$ and $q_{c}$ are the heat exchanged with the hot disc, respectively by radiation and contact, and $q_{i}$ is the conduction heat transferred from the inner surface to the driven spindle. $F_{H-C}$, which accounts for the fraction of radiation energy leaving the hot disc and reaching the cold disc, is the so-called view factor and was given the value of 0.26 as suggested by Juul [20] for similar configurations.

Moreover, looking at the radiation heat exchanges with the environment, the Stefan-Boltzmann law for radiation was employed. This is clearly non-linear; therefore, linearization was performed to further simplify the problem by introducing an equivalent convective coefficient, $h_{r H B}$, and an equivalent radiation temperature $u_{H D}{ }^{*}$. For the heat exchanged with the hot disc, $q_{r H D}$ these coefficients were obtained by least mean square minimization. This allowed to analytically solving the mathematical problem (Figure 3):

$$
q_{r H D}=\varepsilon \sigma\left(u^{4}-u_{H D}{ }^{4}\right)=h_{r H B}\left[u-u_{H D}{ }^{*}\right],
$$

where $\varepsilon$ is the emissivity and $\sigma$ is the Stefan-Boltzmann constant. The radiation with the surrounding environment $\left(q_{r R}\right)$ was treated in the same way.

In Eq. ( 3 ), $q_{c}$ is the heat flux at the contact arc computed as follows:

$$
q_{c}=h t c\left(\bar{u}-u_{H D}\right)
$$

where $\bar{u}$ and htc were considered respectively as the mean surface temperature along the contact arc and the heat transfer coefficient.

The contact arc segment of opening $\varphi_{0}$ was estimated through Hertz theory for the line contact without taking into account any material plastic behavior, hence underestimating its value, especially for large loads, when plasticity may significantly affect the contact area. However, it should be noted that since $\varphi_{0}$ is usually extremely small (less than $1^{\circ}$ ), the estimation of the heat flux $q_{c}$ by means of temperature measurements far from the contact zone, where the effect of the flash temperature is negligible [21], is almost entirely unaffected by any error in the determination of $\varphi_{0}$.

In Eq. ( 6 ), $\bar{u}$ is approximated by superimposing a two-dimensional quasi steady-state solution for the angular temperature variation due to the concentrated heat flux to the one-dimensional solution, which evaluates the instantaneous evolution of the average surface temperature.

After having introduced and explained all the terms in Eq. ( 3 ), this partial differential equation subjected to the non-homogeneous boundary conditions in Eqs. (3) and (4) can be solved. The general solution is given by:

$$
u(r, t)=u_{S S}(r)+\sum_{m=1}^{+\infty} A_{m} R\left(\lambda_{m} r\right) e^{-\alpha \lambda_{m}^{2} t},
$$


where $u_{S S}(r)$ is the solution of the associated steady-state problem , $A_{m}$ is the generalized Fourier coefficient which satisfies the initial condition, $R\left(\lambda_{m} r\right)$ is the solution of the Bessel equation and $\lambda_{m}$ is the eigenvalue which solves the associated Sturm-Liouville problems [22].

\subsection{D quasi steady-state solution}

Considering that the effect of concentrated heat fluxes is to locally raise the surface temperature in the form of a flash temperature, the determination of the mean surface temperature along the circumference and the mean surface temperature, $\bar{u}$, along the contact arc requires solving the heat transfer problem in the circumferential direction; this can be achieved by solving a two-dimensional problem for which an analytical solution is sought.

A suitable evaluation of the flash temperature, namely the temperature reached locally under the effect of a moving band-shaped heat source, was in this case approached through the quasi steady-state solution for a rotating infinite hollow cylinder subjected to a fixed generic concentrated heat flux on the external surface [23].

Under steady-state conditions, Eq. ( 1 ) becomes:

$$
\frac{1}{r} \frac{\partial}{\partial r}\left(r \frac{\partial u}{\partial r}\right)-\frac{\omega}{\alpha} \frac{\partial u}{\partial \theta}+\frac{1}{r^{2}} \frac{\partial^{2} u}{\partial \theta^{2}}=0
$$

Following the quasi steady-state approach, Eq. (8) was solved by applying the following general timedependent boundary condition for the external concentrated band-shaped heat source $q_{e}$ :

$$
-k \frac{\partial}{\partial r} u\left(R_{e}, t\right)=\left\{\begin{array}{cc}
q_{e} & -\varphi_{0} / 2 \leq \theta \leq \varphi_{0} / 2 \\
h_{e}\left[u\left(R_{e}, \theta\right)-u_{e}\right] & \varphi_{0} / 2 \leq \theta \leq 2 \pi-\varphi_{0} / 2
\end{array}\right.
$$

where $h_{e}$ isthe generic convection coefficient with the surrounding environment and $u_{e}$ the environment temperature.

Using the integral Fourier transform [24]:

$$
\tilde{u}(r, n)=\int_{-\pi}^{\pi} u(r, \theta) e^{-j n \theta} d \theta
$$

Eq. (8) can be transformed into a particular case of the Bessel equation:

$$
r^{2} \frac{d^{2} \widetilde{u}}{d r^{2}}+r \frac{d \widetilde{u}}{d r}+\left(-j \frac{n \omega}{\alpha} r^{2}-n^{2}\right) \tilde{u}=0
$$

Given the narrow opening of the contact $\operatorname{arc}, \varphi_{0}$, the problem was solved under the following boundary condition, where the heat transfer due to convection with the environment is applied over the entire angular range $-\pi \leq \theta \leq \pi$, namely along the entire disc circumference ([24]-[26]):

$$
\frac{d}{d r} \tilde{u}\left(R_{e}, n\right)=-H_{e} \tilde{u}\left(R_{e}, n\right)+\widetilde{K}_{e_{n}}
$$

with:

$$
H_{e}=h_{e} / k
$$




$$
\widetilde{K}_{e_{n}}=\left(h_{e} \int_{-\pi}^{\pi} u_{e} e^{-j n \theta} d \theta+\int_{-\varphi_{0} / 2}^{\varphi_{0} / 2} q_{e} e^{-j n \theta} d \theta\right) / k
$$

The solution of Eq. ( 11$)$ is a composition of Bessel functions of first $\left(J_{n}\right)$ and second kind $\left(Y_{n}\right)$ of order $n$ with imaginary argument $\epsilon_{n}=\sqrt{-j \frac{n \omega}{\alpha}}$ :

$$
\tilde{u}(r, n)=D_{n} J_{n}\left(\epsilon_{n} r\right)+E_{n} Y_{n}\left(\epsilon_{n} r\right)
$$

Finally, by applying the Inverse Fourier Transform, the quasi steady-state solution turns into in the following discrete representation [27]:

$$
u(r, \theta)=\frac{1}{2 \pi} \sum_{n=0}^{+\infty} \gamma_{n} \operatorname{Re}\left[\left(D_{n} J_{n}\left(\epsilon_{n} r\right)+E_{n} Y_{n}\left(\epsilon_{n} r\right)\right) e^{j n \theta}\right],
$$

where $\gamma_{n}=\left\{\begin{array}{ll}1 & n=0 \\ 2 & n \neq 0\end{array}\right.$.

Hence, the quasi steady-state solution in discrete representation can be re-written as follows:

$$
u(r, \theta)=u_{S S}(r)+\frac{1}{\pi} \sum_{n=1}^{+\infty} \operatorname{Re}\left[\left(D_{n} J_{n}\left(\epsilon_{n} r\right)+E_{n} Y_{n}\left(\epsilon_{n} r\right)\right) e^{j n \theta}\right]
$$

where $u_{S S}(r)$ is the solution for $n=0$, which is basically the steady-state solution for the equivalent onedimensional problem, and the summation corresponds to the effect of the axisymmetric thermal load. Thus, the first term represents the average surface temperature, whereas the second one describes the angular variation of temperature due to the concentrated heat flux.

\subsection{Boundary conditions to mimic the specific testing configuration}

Referring to the testing configuration reported in Figure 1, the boundary conditions in Eq. ( 9 ) become:

$$
-k \frac{\partial}{\partial r} u\left(R_{e}, \theta, t\right)=\left\{\begin{array}{cc}
q_{c} & -\varphi_{0} / 2 \leq \theta \leq \varphi_{0} / 2 \\
h_{a}\left[u-u_{a}\right]+h_{r R}\left[u-u_{r R}{ }^{*}\right]+F_{H-C} h_{r H D}\left[u-u_{H D}{ }^{*}\right] & -\pi \leq \theta<\pi
\end{array}\right.
$$

where $h_{a}, h_{r R}, u_{a}$ and $u_{r R}{ }^{*}$ are respectively the convective heat transfer coefficient, the equivalent convective coefficient for radiation, the air temperature and the equivalent radiation temperature.

The parameters to be used in conjunction with Eq. (12) to achieve the bi-dimensional solution for the mathematical problem in Fourier transform space can now be written as:

$$
\begin{gathered}
H_{e}=\left(h_{a}+h_{r R}+F_{H-C} h_{r H D}\right) / k \\
\widetilde{K}_{e_{n}}=\left(\int_{-\pi}^{\pi}\left(h_{a} u_{a}+h_{r R} u_{r R}{ }^{*}+F_{H-C} h_{r H D} u_{H D}{ }^{*}\right) e^{-j n \theta} d \theta+\int_{-\varphi_{0} / 2}^{\varphi_{0} / 2} q_{c} e^{-j n \theta} d \theta\right) / k
\end{gathered}
$$

Since a test starts when a constant external surface temperature is achieved on the cold disc (steady-state condition), the mathematical description captures this aspect. Hence, an initial temperature field, depending on the radial coordinate, was applied to the one-dimensional transient solution. The initial condition corresponds to the solution of a one-dimensional steady-state heat conduction problem applied to a hollow 
cylinder, where the external surface is kept to a constant temperature $\left(T_{e}\right)$ and the internal surface is subjected to an internal heat flux, coming from the inverse analysis solution of $\left(-k \frac{\partial}{\partial r} u\left(R_{i}, t\right)=q_{i}\right)$. Thus, the initial condition is:

$$
u(r, t=0)=T_{e}+q_{i} R_{i}\left(\ln R_{e}-\ln r\right) / k
$$

\subsection{Inverse analysis procedure}

The object of the inverse analysis was the heat transfer solution applied to the determination of the boundary conditions, where the parameter to be estimated is the heat transfer coefficient htc. The optimum solution was achieved through a minimization process, whereby the mismatch between the computed temperature and the measured temperature was minimized by solving a least squares problem. Different approaches are available in the literature; although the most commonly used is the Levenberg-Marquard method [28], the Trust-Region algorithm [29] was chosen to achieve the solution in a limited number of iterations. This is due to the fact that this method employs linear algebra that does not require to store and/or operate on full matrixes. A comprehensive description of the applied optimization algorithm can be found in [30]. The heat transfer coefficient $h t c$ was determined and the heat rate during the test was calculated taking into account the opening of the contact $\operatorname{arc} \varphi_{0}$.

The inverse analysis returned also an estimated value for the average heat flux, $q_{i}$, exchanged with the spindle. As mentioned above, the contributions of radiation are computed by the linearized law defined in Eq. (5), whereas convection heat transfer is estimated through empirical correlations available from the literature [31].

\section{Results and discussion}

\subsection{Evolution of the surface temperature at the measuring point}

In Figure 4 measurements of the surface temperature evolution during the contact step are shown for the cases investigated here. Specifically, the temperature increase with respect to the starting surface temperature is reported as a function of time. In all cases, a temperature raise was clearly detectable, meaning that the heat fluxes received by contact and radiation from the hot disc were higher than the heat fluxes exchanged with the environment. Increasing both temperature difference between hot and cold disc and normal load, the temperature rise becomes more evident. Moreover, the time evolution plots revealed that 30 minutes of contact between the two rings were sufficient to approach a steady state condition with small temperature variations.

\section{2 htc and $Q_{i}$ calibration by inverse analysis}

Firstly, the analytical solution for the evolution of the surface temperature in the measurement area was calibrated to match the surface temperature instantaneously measured; this was achieved by acting on the parameters $h t c$ and $Q_{i}$ (where $Q_{i}=q_{i} \cdot A_{i}$ and $A_{i}$ is the internal area). 
A dedicated inverse analysis was run for each case. Since the inverse analysis algorithm does not distinguish between a global or a local minimum, four different starting points were considered to check that the global minimum was obtained; therefore, four different analyses were run for each experiment. Starting points were chosen in order to investigate a wide range of values and they all converged to the same minimum, which corresponded to the same problem solution. As example, the results of the inverse analysis for the fourth case reported in Table 2 are shown in Figure 5.

An example of the matching between the predicted trends and the measured ones is shown in Figure 6 . The analytical model fits the experimental data with a maximum error which is lower than $10{ }^{\circ} \mathrm{C}$ and a sufficient accuracy in estimating the heat fluxes.

Moreover, the quasi steady-state solution for the temperature distribution along the disc circumference confirmed the validity of the basic assumptions according to which the temperature measured at $90^{\circ}$ from the contact region is almost unaffected by the very fast temperature increase (flash event) occurring at the contact arc (Figure 7).

Calculated values of $h t c$ and $Q_{i}$ are reported for all considered experimental conditions in Table 2. High values of $h t c$ and $Q_{i}$ correspond to high temperature differences between the discs. Small variations of $h t c$ and $Q_{i}$ were observed when the normal load was changed from $500 \mathrm{~N}$ to $2000 \mathrm{~N}$ (respectively $136 \mathrm{MPa}$ and $272 \mathrm{MPa}$ of average Hertzian pressure at room temperature).

\begin{tabular}{ccccc}
\hline Case & \multirow{2}{*}{ Normal load } & Initial temperature difference & htc $\left[\mathrm{W} / \mathbf{m}^{2} \mathrm{~K}\right]$ & $\boldsymbol{Q}_{\boldsymbol{i}}[\mathrm{W}]$ \\
\hline $\mathbf{1}$ & \multirow{2}{*}{$500 \mathrm{~N}$} & $220^{\circ} \mathrm{C}$ & 11160 & 5.93 \\
$\mathbf{2}$ & \multirow{2}{*}{$2000 \mathrm{~N}$} & $460^{\circ} \mathrm{C}$ & 21700 & 20.36 \\
$\mathbf{3}$ & $220^{\circ} \mathrm{C}$ & 9757 & 3.25 \\
\hline
\end{tabular}

Table 2: Calculated values of htc and $q_{i}$ for all considered experimental conditions.

\subsection{Contact heat-transfer rate}

The contact heat-transfer rate $\left(Q_{c}=A_{c} \cdot q_{c}\right.$, where $A_{c}$ is the contact area) is expected to decrease with time according to Eq. (6) because the surface temperature of the cold disc increases during testing. This is confirmed in Figure 8, where the calculated $Q_{c}$ evolution is plotted during the contact step, together with the experimental cold disc surface temperature. In the same graph, the heat-transfer rate balance, namely the integral of all heat-transfer rates to which the cold sample was subjected, is plotted. Its value goes to zero in approaching the final stage of the test, when a steady state condition is achieved.

The results of the four experimental cases were finally compared in terms of the steady-state heat-transfer rate, $Q_{c}$ (Figure 9). Both the effects of normal load and temperature difference between discs under steady condition were examined. It is clearly shown that the temperature difference between the discs has a stronger effect on the system response than the applied normal load. When the latter parameter is increased by a factor of $4, Q_{c}$ is only 1.5 times greater, whereas an increase of the temperature difference of about 1.6 times, for a given normal load, leads to an increase of the contact heat transfer rate by factor of 3-4. 


\section{Conclusions}

A comprehensive methodology to investigate heat flux in a ring-on-ring tribometer was developed. Thermal fluxes under high contact pressures and temperature differences were assessed by an experimental campaign, corroborated by a robust procedure of inverse analysis based on an innovative analytical solution of the underlying heat transfer problem. The proposed techniques were applied to surface temperature measurements carried out remotely from the contact area. This procedure allowed for accurate quantitative predictions of the heat transfer coefficient and heat transfer rates.

Through long-term tests, carried out under pure rolling conditions using an ad hoc developed tribometer, contact heat-transfer rates were evaluated and reported as a function of normal load and steady-state temperature differences. Results show that, within the range of parameters explored in the present study, the temperature differences between test components have a much larger influence on the heat transfer rate than the variation of the applied normal load.

This innovative methodology can be applied to estimate the heat fluxes between contact surfaces in operating conditions similar to those occurring in real industrial hot deformation processes such as hot rolling.

\section{Acknowledgments}

Mario Rossi, director of TenarisDalmine R\&D center, is kindly acknowledged for the permission to publish this paper. The authors wish to thank Ettore Anelli, principal scientist at TenarisDalmine, for helpful discussions and suggestions. Authors F.A. and E.R. acknowledge support from "F.Y.RE. - Fostering Young REsearchers project" by Fondazione Cariplo at the University of Bergamo. 


\section{References}

[1] Lenard J.G. (2000). Tribology in metal rolling. Keynote presentation forming Group F. CIRP Annals Manufacturing Technology, 49(2): 567-590.

[2] Devadas C., Samarasekera I.V., Hawbolt E.B. (1991). The thermal and metallurgical state of steel strip during hot rolling: Part I. Characterization of heat transfer. Metallurgical Transactions A, 22(2): 307-319.

[3] Lambert M.A., Fletcher L.S. (1997). Review of models for thermal contact conductance of metals. Journal of Thermophysics and Heat Transfer, 11(2): 129-140.

[4] Jackson R.L., Ghaednia H., Elkady Y.A., Bhavnani S.H., Knight R.W. (2012). A closed-form multiscale thermal contact resistance model. Components, Packaging and Manufacturing Technology, IEEE Transaction, 2(7): 1158-1171.

[5] Thompson M.K., Thompson J.M. (2010). Considerations for the incorporation of measured surfaces in finite element models. Scanning, 32(4): 183-198.

[6] Yovanovich M.M. (2005). Four decades of research on thermal contact, gap, and joint resistance in microelectronics. Components and Packaging Technologies, IEEE Transactions, 28(2): 182-206.

[7] Hlady C.O., Brimacombe J.K., Samarasekera I.V., Hawbolt E.B. (1995). Heat transfer in the hot rolling of metals. Metallurgical and Materials Transactions B, 26(5): 1019-1027.

[8] Huang C., Ju T., Tseng A. (1995). The estimation of surface thermal-behavior of the working roll in hotrolling process. International Journal Of Heat And Mass Transfer, 38(6): 1019-1031.

[9] Zhang W., Bay N. (1997). Numerical analysis of cross shear plate rolling. Annals of the CIRP, 46(1): 195200.

[10] Keanini K. (1998). Inverse estimation of surface heat flux distributions during high speed rolling using remote thermal measurements. International Journal of Heat and Mass Transfer, 41(2): 275-285.

[11] Saboonchi A., Abbaspour A.M. (2004). Changing the geometry of water spray on milling work roll and its effect on work roll temperature. Journal of Materials Processing Technology, 148(1): 35-49.

[12] Montmitonnet P. (2006). Hot and cold strip rolling processes. Computer Methods in Applied Mechanics and Engineering, 195(48-49): 6604-6625.

[13] Volle F., Maillet D., Gradeck M., Kouachi A., Lebouche M. (2009). Practical application of inverse heat conduction for wall condition estimation on a rotating cylinder. International Journal of Heat and Mass Transfer, 52(1-2): 210-221. 
[14] Chen W.L., Yang Y.C. (2010). Inverse problem of estimating the heat flux at the roller/workpiece interface during a rolling process. Applied Thermal Engineering, 30(10): 1247-1254.

[15] Gradeck M., Kouachi A., Borean J., Gardin P., Lebouche M. (2011). Heat transfer from a hot moving cylinder impinged by a planar subcooled water jet. International Journal of Heat and Mass Transfer, 54(25-26): 5527-5539.

[16] Weisz-Patrault D., Ehrlacher A., Legrand N. (2012). Evaluation of temperature field and heat flux by inverse analysis during steel strip rolling. International Journal of Heat and Mass Transfer, 55(4): 629-641.

[17] Beynon J.H. (1999). Finite-element modelling of thermomechanical processing. Philosophical Transactions of the Royal Society of London Series A, 357(1756): 1573-1587.

[18] Saunders N., Guo Z., Li X., Miodownik A.P., Schillé J.-P. (2003). Using JMatPro to Model Materials Properties and Behavior. Journal of The Minerals, Metals \& Materials , 55(12): 60-65.

[19] Yevtushenko A., Chapovska R. (1997). Distribution of the friction heat between two stationary pins and a rotating disc. Heat and Mass Transfer, 33(1-2): 59-65.

[20] Juul N.H. (1982). View factors in radiation between two parallel oriented cylinders of finite length. Journal of Heat Transfer, 104(2): 384-388.

[21] Block H. (1963). Flash temperature concept. Wear, 6(6): 483-494.

[22] Hahn D.W., Necati Ozisik M. (2012). Heat Conduction, third Edition. John Wiley \& Sons, Inc., Hoboken, New Jersey.

[23] Patula E.J. (1981). Steady-state temperature distribution in a rotating roll subject to surface heat fluxes and convective cooling. Journal of Heat Transfer, 103(1): 36-41.

[24] Gecim B., Winer W. (1984). Steady temperature in a rotating cylinder subject to surface heating and convective cooling. Journal of Tribology, 106(1):120-126.

[25] Alilat N., Baïri A., Laraqi N. (2004). Three-dimensional calculation of temperature in a rotating disk subject to an eccentric circular heat source and surface. Numerical Heat Transfer, Part A, 46(2): 167-180.

[26] Osman T., Guenoun S., Boucheffa A. (2010). Temperature field in a rotating roller subjected to interface heating. The European Physical Journal Applied Physics, 50(2): 1-12.

[27] Sneddon I.N. (1972). The Use of Integral Transform. McGraw-Hill, New York.

[28] Necat Ozisik M., Orlande H.R.B. (2000). Inverse Heat Transfer: Fundamentals and Applications. Taylor \& Francis Inc., New York. 
[29] Steihaug T. (1983). The conjugate gradient method and trust regions in large scale optimization. SIAM Journal on Numerical Analysis, 20: 626-637.

[30] Arizzi F., Rizzi E. (2014). Elastoplastic parameter identification by simulation of static and dynamic indentation tests. Modelling and Simulation in Materials Science and Engineering, 22(3): 035017.

[31] Holman F.P. (2010). Heat Transfer, tenth Edition. McGraw Hill, New York. 
Figure 1: Schematic of the testing configuration and temperature measurement.

Figure 2: Rotating hollow cylinder under external heat conduction source and surrounding external and internal convection.

Figure 3: Stefan-Boltzmann law vs. linearized radiation law $\left(h_{r H D}=62 \mathrm{~W} /{ }^{\circ} \mathrm{Cm}^{2}\right.$ and $\left.u_{H D}{ }^{*}=1215{ }^{\circ} \mathrm{C}\right)$ in cold disc temperature range.

Figure 4: Experimental temperature increase evolution for different test conditions.

Figure 5: Inverse analysis convergence curves (Case 4: $2000 \mathrm{~N} / 800^{\circ} \mathrm{C}$ ).

Figure 6: Comparison between calculated and experimental curves (Case 4: $2000 \mathrm{~N} / 800^{\circ} \mathrm{C}$ ).

Figure 7: Example of calculated surface temperature variation along the cold disc circumference (Case $\left.4: 2000 \mathrm{~N} / 800^{\circ} \mathrm{C}\right)$.

Figure 8: Heat-transfer rate variation during testing (Case 4: $2000 \mathrm{~N} / 800^{\circ} \mathrm{C}$ ). Evolution of surface temperature of the cold disc and heat rate balance are also shown.

Figure 9: Final contact heat-transfer rate, $Q_{c}$, for various normal loads and steady-state temperature difference between discs. 


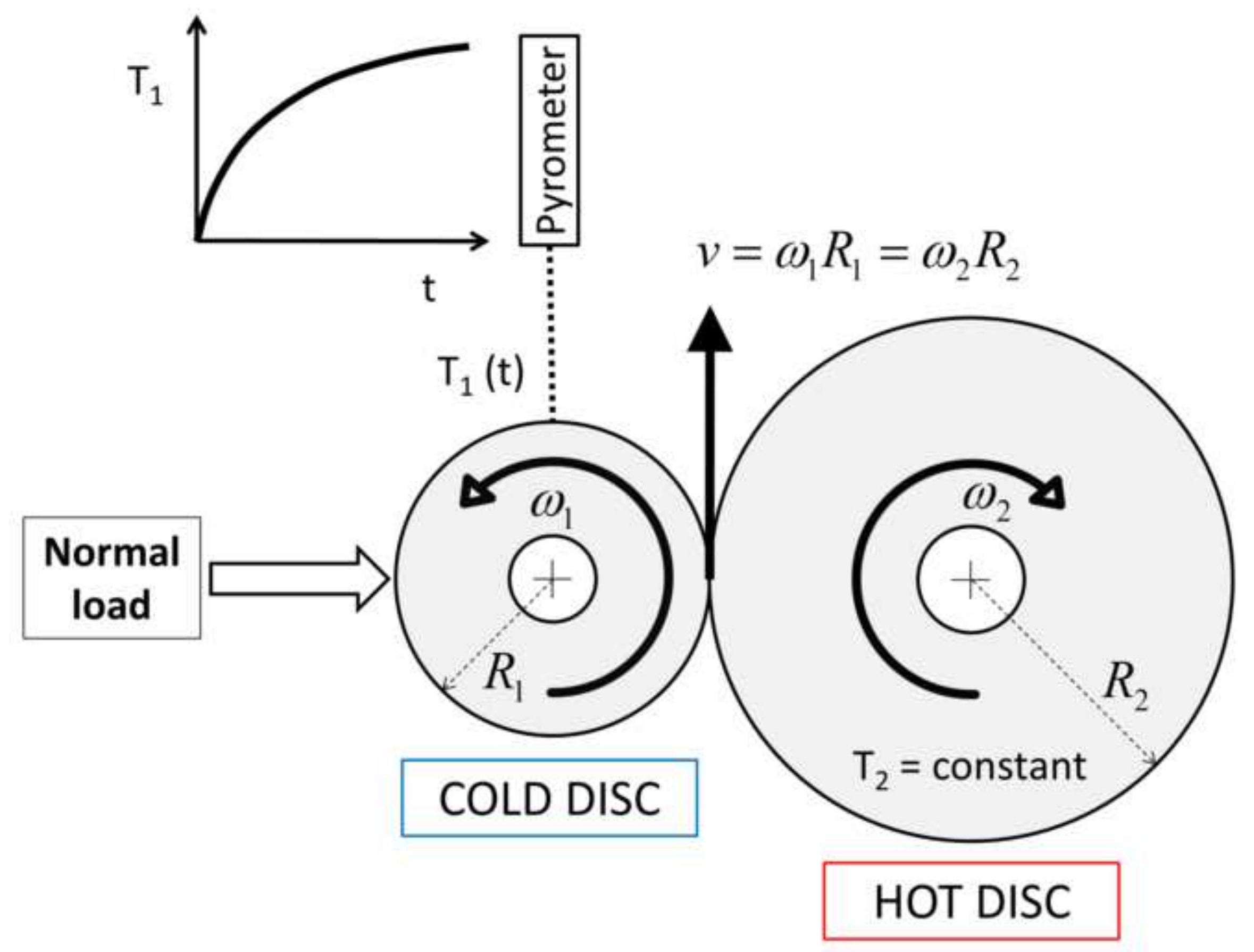




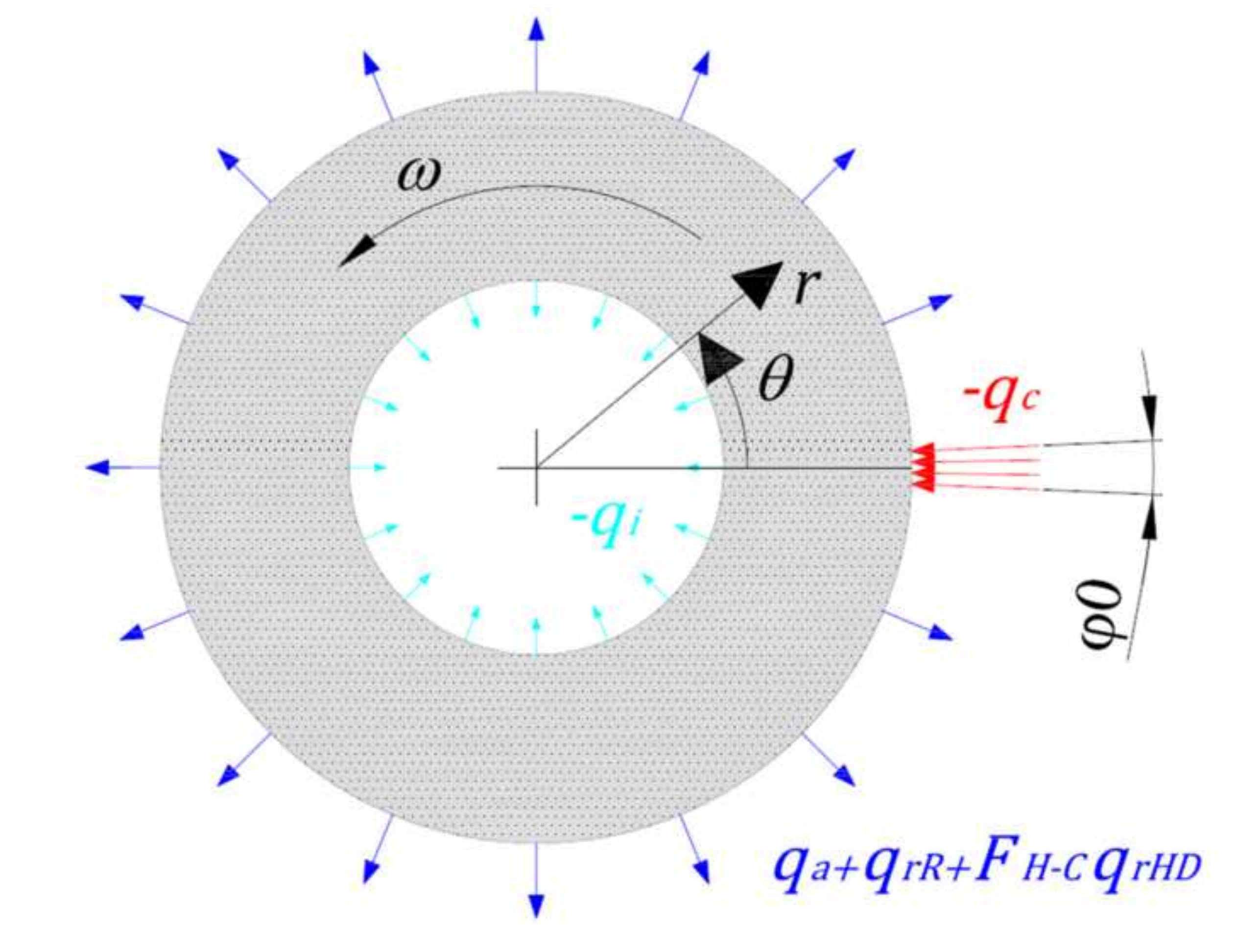

.

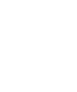

(1)
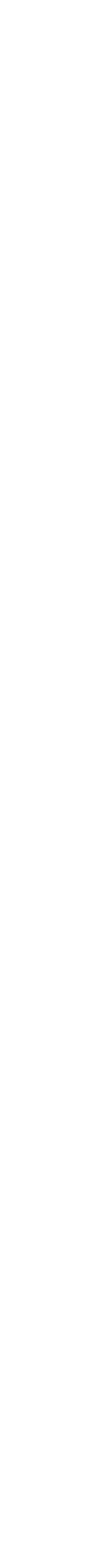

d
$\mathbf{d}$
$\mathbf{d}$ 


\section{Hot disc temperature $=800{ }^{\circ} \mathrm{C}$}

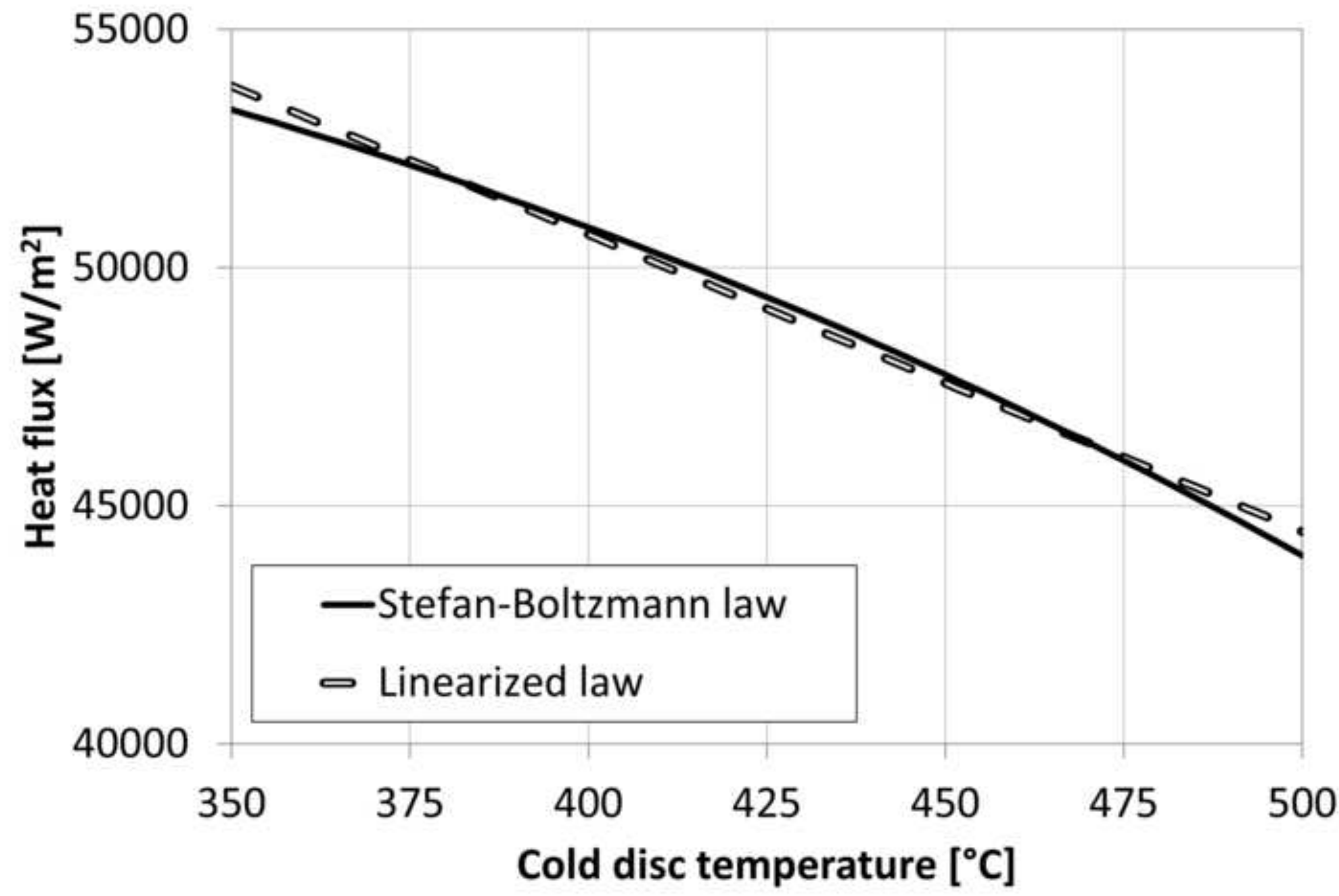




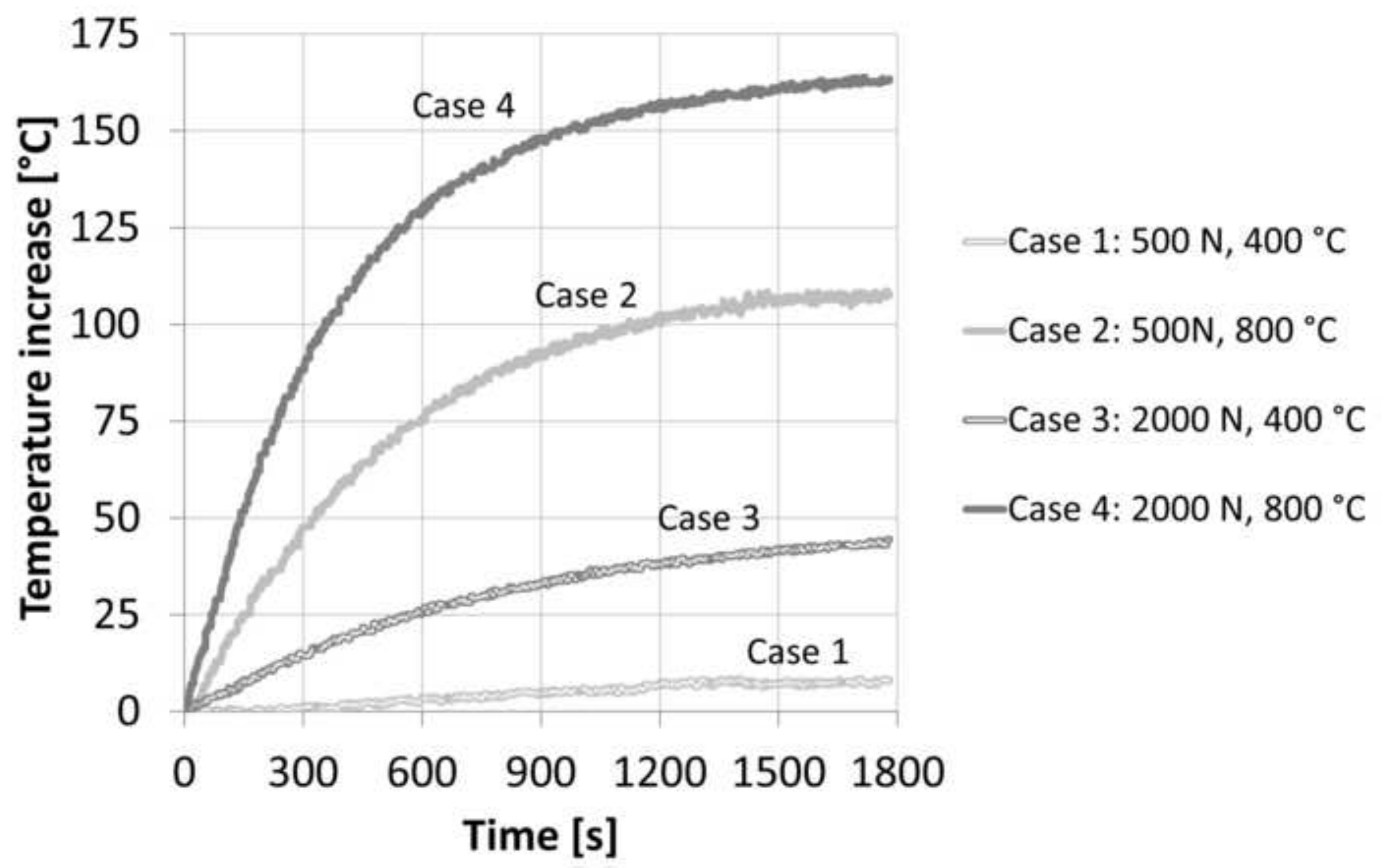


Figure 5

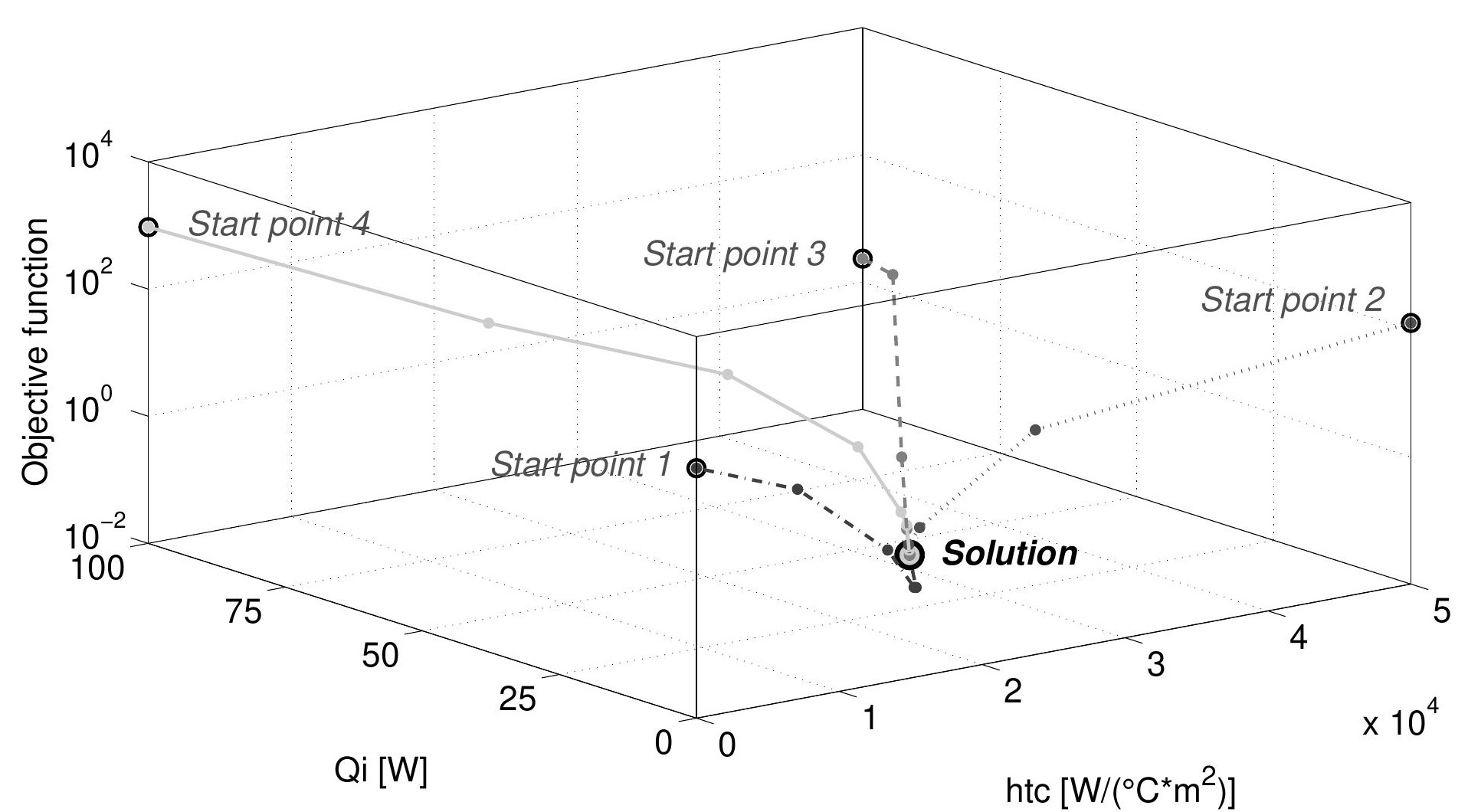

Figure

5

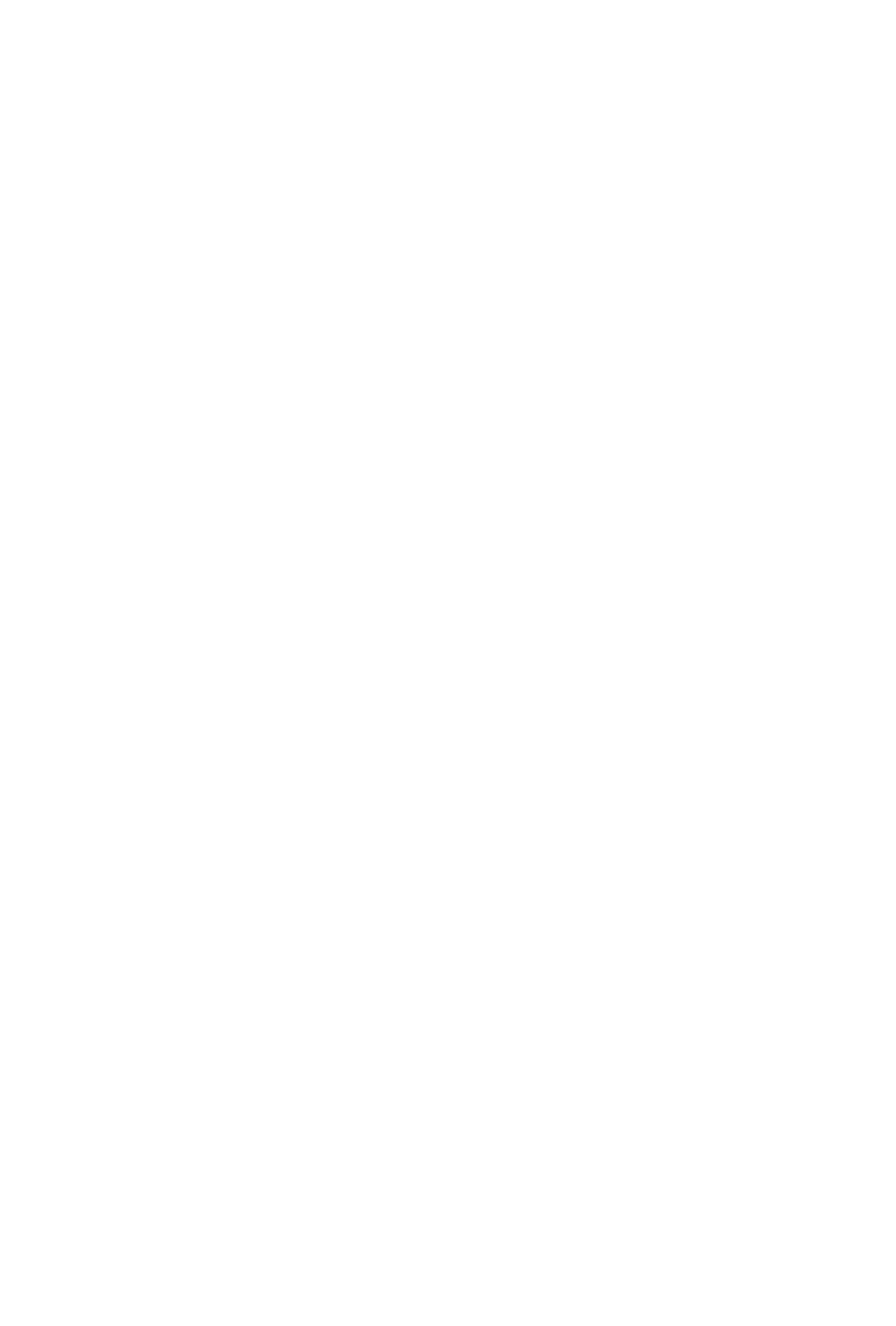
htc $\left[\mathrm{W} /\left({ }^{\circ} \mathrm{C}^{*} \mathrm{~m}^{2}\right)\right]$ 
Figure 6

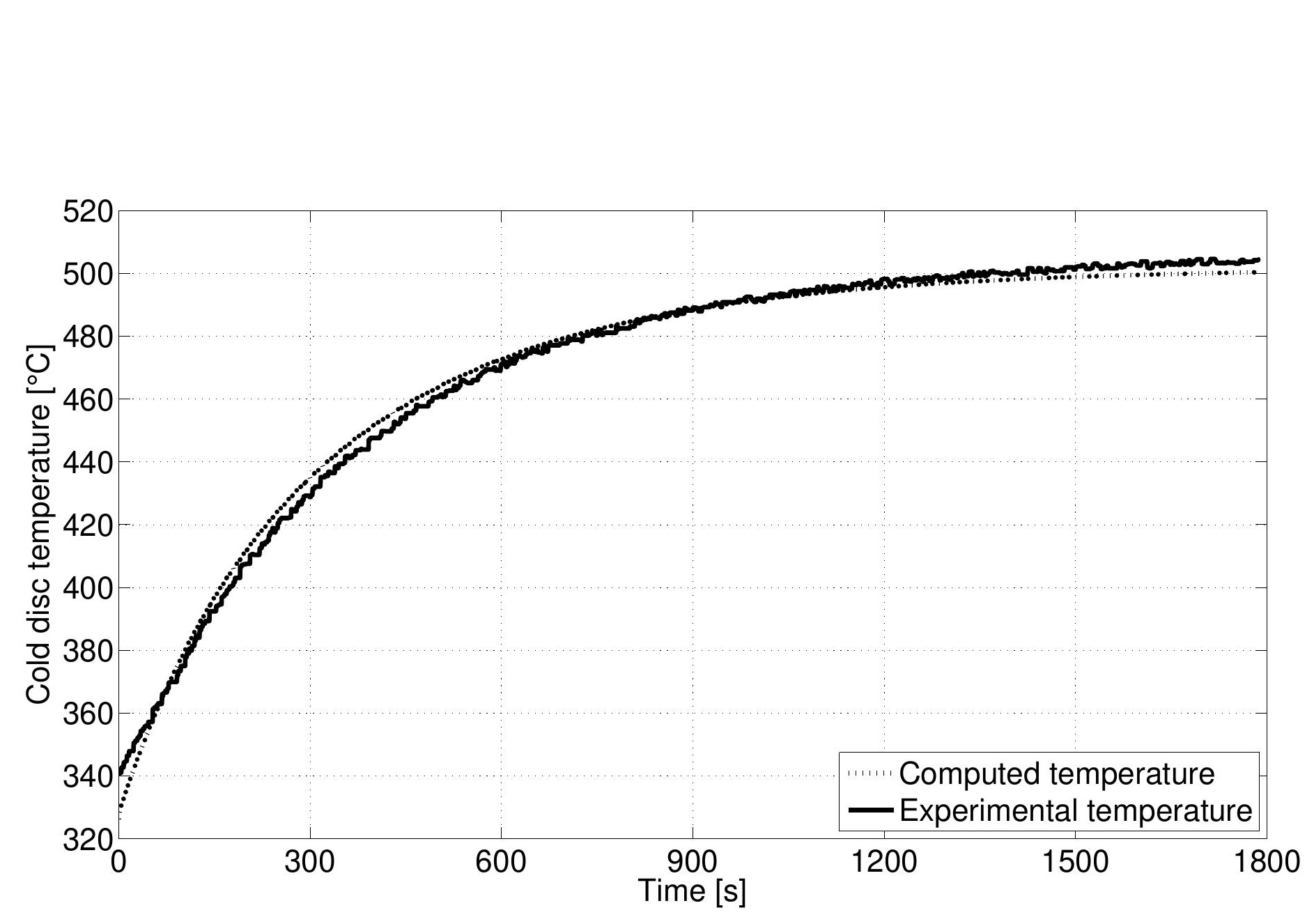




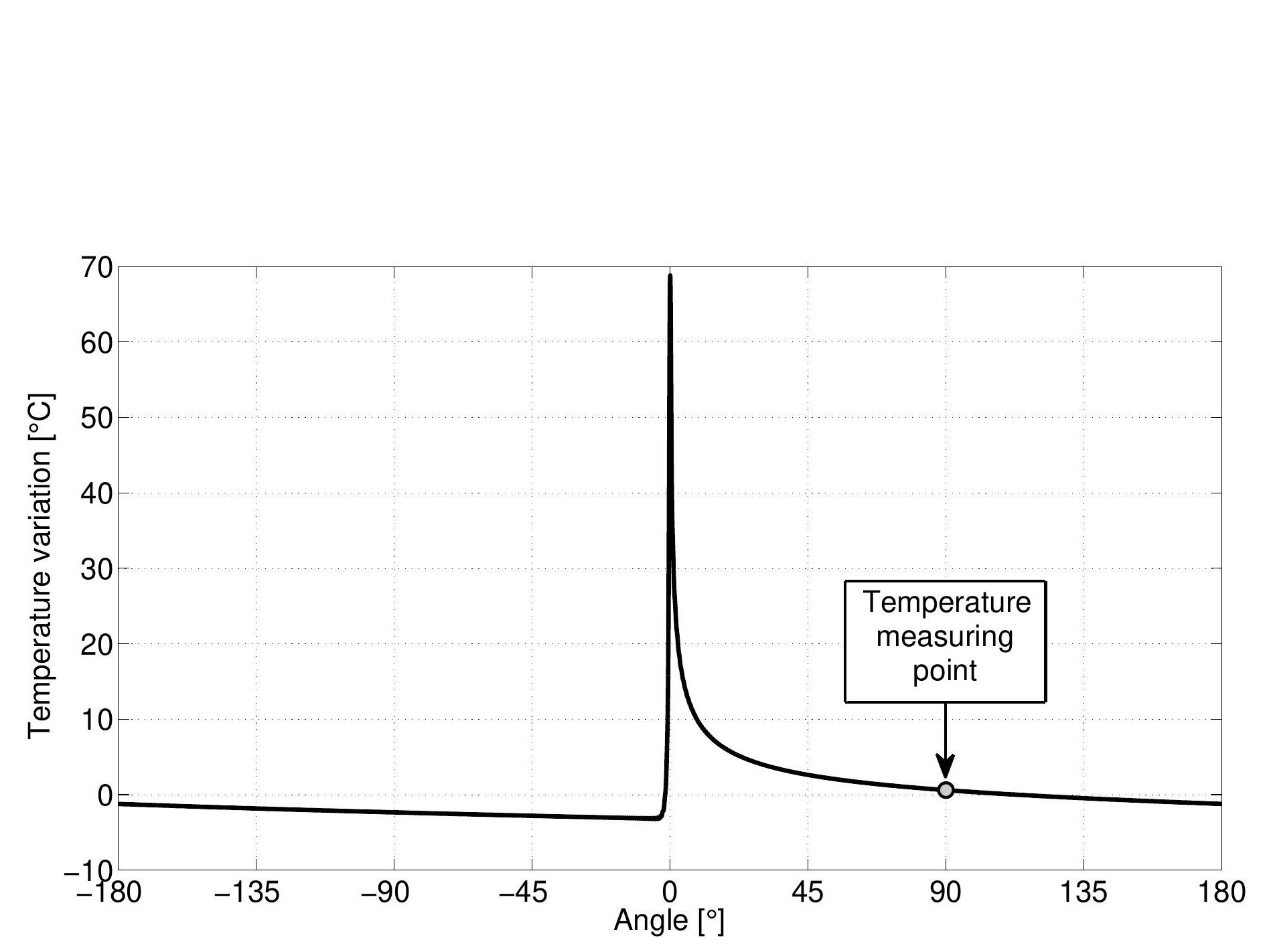

Figure 7

\section{Figure}

7

7
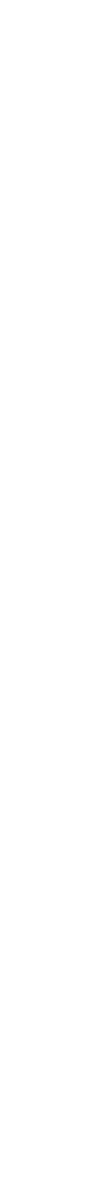

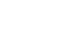
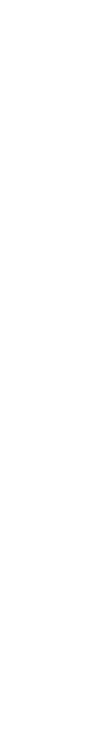


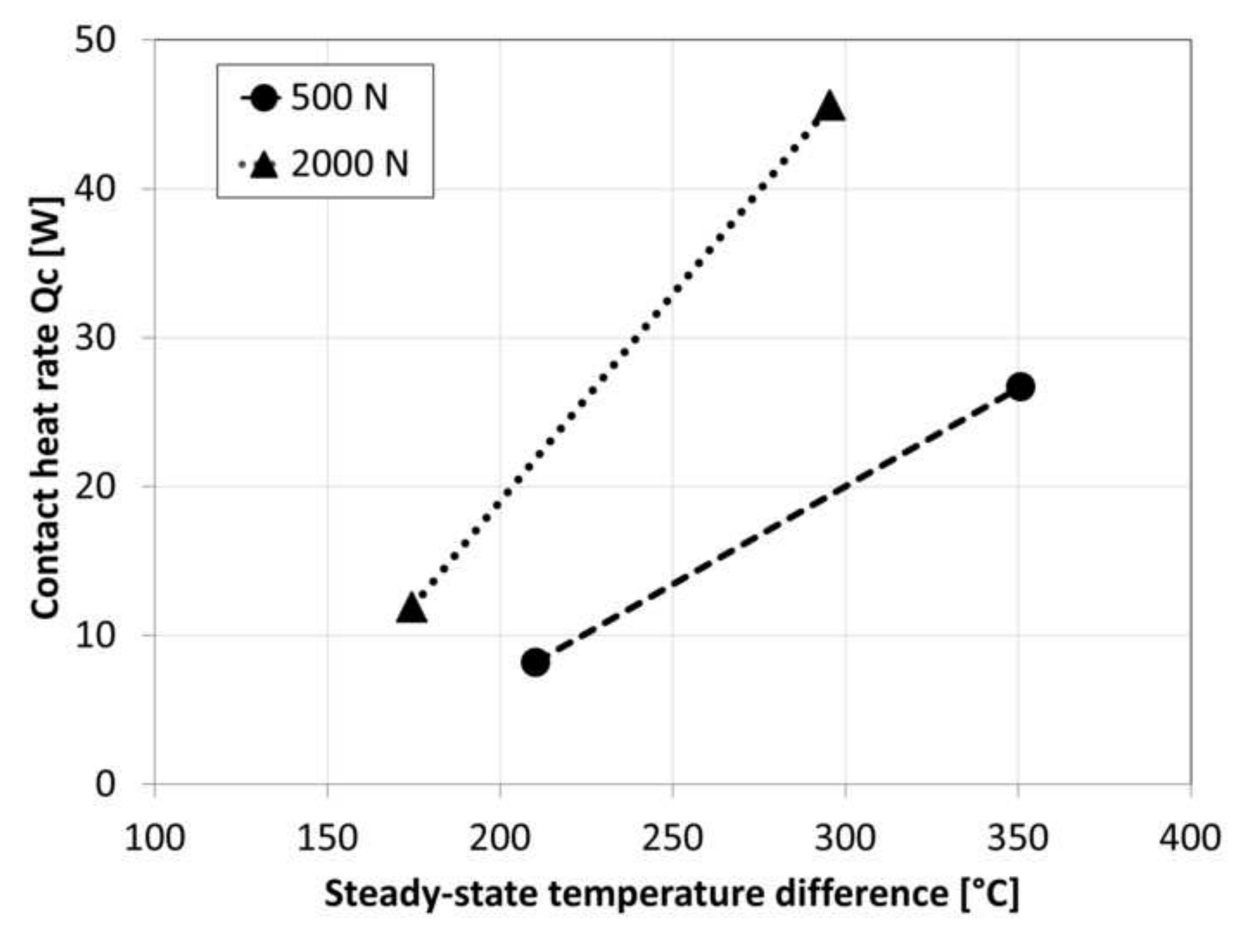
. .

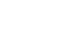

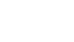

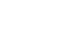
.

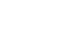

